\title{
Rapid Communication - Effect of Exercise Training on Asymmetric Dimethylarginine Concentration in Women Aged 65-74 years with Type 2 Diabetes
}

\author{
Kevin R Serre ${ }^{1 *}$, Michael J. Simmonds ${ }^{2}$, Surendran Sabapathy ${ }^{2}$, Clare L. Minahan ${ }^{2}$ and Gregory C. Gass ${ }^{1,3}$ \\ ${ }^{1}$ Faculty of Health Sciences and Medicine, Bond University, Queensland, Australia \\ ${ }^{2}$ School of Physiotherapy and Exercise Science, Griffith University, Queensland, Australia \\ ${ }^{3}$ Department of Health, Nutrition, and Exercise Sciences, North Dakota State University, North Dakota, USAI
}

\begin{abstract}
Aims/hypothesis: Basal plasma concentration of asymmetric dimethylarginine (ADMA), an endogenous, competitive inhibitor of nitric oxide synthase, is elevated in patients with type 2 diabetes. ADMA may contribute to the endothelial dysfunction and associated vascular complications observed in individuals with type 2 diabetes. The present study investigated the effect of 12 weeks of supervised walking exercise on plasma ADMA concentration in women aged 65-74 years with type 2 diabetes.
\end{abstract}

Materials and methods: Fourteen women (aged $69 \pm 3$ yrs) with uncomplicated type 2 diabetes, completed 12 weeks of supervised, moderate-intensity walking at an intensity equivalent to their individual gas-exchange threshold. Blood was sampled for ADMA concentration before and after a 6-week intervention-free control period, and again after 6 and 12 weeks of exercise training.

Results: Plasma ADMA concentration was found to be significantly lower after 12-weeks of exercise training when compared with baseline ( $0 \mathrm{wk})$ measurements. These results were accompanied by significant increases in time to exhaustion, relative and absolute peak oxygen uptake, and oxygen uptake at gas-exchange threshold.

Conclusion/interpretation: Regular, moderate-intensity exercise decreases circulating ADMA concentrations in older women with type 2 diabetes. These results suggest that ADMA may play a role in the training-induced reduction in cardiovascular disease risk seen with exercise training in individuals with type 2 Diabetes.

Keywords: Exercise training; Type 2 diabetes; Asymmetrical dimethylarginine

Abbreviations: ADMA: Asymmetric dimethylarginine; BMI: Body Mass Index; BP: Blood Pressure; CVD: Cardiovascular Disease; FEV $_{1}$ : Forced Expiratory Volume In 1 Second; FVC: Forced Vital Capacity; $\mathrm{HbA}_{1 c}$ : Glycated Hemoglobin; NO: nitric oxide; TE: Time To Exhaustion; $\mathrm{T}_{\mathrm{ge}}$ : gas-exchange threshold; $\mathrm{V}_{\mathrm{E}}$ BTPS: Expired Minute Ventilation; $\mathrm{VO}_{2}$ peak: Peak Oxygen Uptake; $\mathrm{VCO}_{2}$ : Carbon Dioxide Output

\section{Introduction}

Type 2 diabetes is associated with a two-to-three fold higher incidence of macrovascular atherosclerotic disease compared to nondiabetic individuals [1]. The increased incidence of microvascular atherosclerotic disease cannot be fully explained by the presence of conventional risk factors such as hypertension, obesity, and dyslipidemia [2], that often accompany diabetes. Decreased nitric oxide (NO) bioavailability, a key modulator of endothelial function, may contribute to the development of the microvascular complications associated with type 2 diabetes [3], which is in part modulated by asymmetric dimethylarginine (ADMA) $[4,5]$.

ADMA is an endogenous, competitive inhibitor of NO synthase and a known independent cardiovascular disease risk factor [6,7]. Plasma ADMA concentrations have been shown to be elevated in individuals with increased cardiovascular disease risk [8-11], older individuals [12], and in patients with type 2 diabetes [4,5,13]. Elevated plasma ADMA concentrations are thought to play a role in endothelial dysfunction and associated vascular complications seen in individuals with type 2 diabetes [14-16]. While regular physical exercise has been shown to improve endothelial function in individuals with type 2 diabetes [17,18], the pathophysiological basis for these exerciseinduced improvements remains unclear.
Regular physical exercise has been shown to reduce circulating levels of ADMA in patients with increased risk of coronary heart disease [19], in patients with metabolic syndrome [20] and type 1 diabetes mellitus [21]. To date, no study has examined the effects of repeated bouts of moderate-intensity exercise on ADMA concentrations in individuals with type 2 diabetes. Therefore, the aim of the present study was to determine the effects of 12 weeks of moderate-intensity exercise on plasma ADMA in women 65-74 years of age with type 2 diabetes.

\section{Methods}

\section{Participants and Recruitment Strategies}

Fifteen women with type 2 diabetes, aged 65-74 years participated. Participants were recruited from local General Medical Practitioner clinics, media advertisements and mail-outs by Diabetes Australia Queensland. Recruitment was difficult from this age group. Approximately 5,000 flyers were distributed to local General Medical Practitioner clinics, diabetes educators, hospitals and pharmacies. Newspaper and radio advertisements were run weekly over a 6-month period. From patient databases and all contacts, approximately 500

*Corresponding author: Kevin R Serre, Faculty of Health Sciences and Medicine, Bond University, Queensland, Australia 4229, Tel: +1 (613) 639 5135; Fax: +1 (613) 383 0156; E-mail: keserre@bond.edu.au

Received November 09, 2011; Accepted November 21, 2011; Published November 23, 2011

Citation: Serre KR, Simmonds MJ, Sabapathy S, Minahan CL, Gass GC (2011) Rapid Communication - Effect of Exercise Training on Asymmetric Dimethylarginine Concentration in Women Aged 65-74 years with Type 2 Diabetes. Endocrinol Metabol Syndrome S5:001. doi:10.4172/2161-1017.S5-001

Copyright: ( 2011 Serre KR, et al. This is an open-access article distributed unde the terms of the Creative Commons Attribution License, which permits unrestricted use, distribution, and reproduction in any medium, provided the original author and source are credited. 
Citation: Serre KR, Simmonds MJ, Sabapathy S, Minahan CL, Gass GC (2011) Rapid Communication - Effect of Exercise Training on Asymmetric Dimethylarginine Concentration in Women Aged 65-74 years with Type 2 Diabetes. Endocrinol Metabol Syndrome S5:001. doi:10.4172/21611017.S5-001

Page 2 of 5

potential participants meeting all eligibility criteria were contacted via mail with help from Diabetes Australia Queensland. After 18 months of extensive recruitment, only 72 persons (14\% of those invited) expressed interest in participating, of which 32 withdrew because of the time commitment needed or because of their unwillingness to exercise. After excluding those who did not meet our inclusion criteria, there was a cohort of 16 persons; with only 15 persons completing all repeated testing periods $(-6,0,6,12 \mathrm{wk})$ and all exercise-training sessions.

Potential participants were initially screened by telephone interview using a standardized set of questions to determine suitability to enter a formal health screening. The following inclusion criteria were used: (i) clinical diagnosis of type 2 diabetes for at least 12 months, (ii) not currently using exogenous insulin (iii) non-smoking, (iv) normal age-relative physical examination, (v) no significant abnormalities in heart rate and rhythm during a graded treadmill exercise test, (vi) no severe musculoskeletal disability and (vii) not currently taking medications known to directly interfere with exercise responses. All participants provided written consent to participate. Each subject's cognitive capacity to give consent was determined using the MiniMental State Examination [22] and assessed by an independent observer. Participants then entered a formal health-screening phase that included anthropometry and pulmonary function testing. Height was measured (in millimeters) using a wall-mounted stadiometer (Harpenden, Holtain Ltd, Crosswell, UK). Body mass was measured using a calibrated digital scale (CH-150k, A \& D Pty Ltd, Thebarton, Australia) and waist and hip circumference were measured. Pulmonary function was assessed (forced vital capacity, forced expiratory volume in 1 second, and forced expiratory volume in 1 second/forced vital capacity ratio) using a calibrated spirometry system (Ultima CPX, Medical Graphics Corporation, St Paul, USA). Supine and standing 12lead EKG (Cardio Perfect, Welch Allyn Inc., Skaneateles Falls, USA) and blood pressure were measured at rest.

After an overnight fast, venous blood samples were drawn (between 0700 - 0830 hours) at an accredited pathology laboratory for glucose, insulin, hemoglobin $\mathrm{A}_{1 c}\left(\mathrm{HbA}_{1 c}\right)$, and homocysteine measurements. Participants were then asked to visit their family physician with the health-screening results and details of the experimental procedures for an opinion about their suitability to participate in the exercise training program. Bond University Human Research Ethics Committee and Griffith University Human Ethics Committee reviewed and approved the experimental protocol.

\section{Determination of Peak Oxygen Uptake and Gas-Exchange Threshold $\left(\mathrm{T}_{\mathrm{ge}}\right)$}

Prior to determining peak oxygen uptake, all participants were thoroughly familiarized with all procedures and equipment. Participants then completed an incremental exercise test to volitional fatigue on a motor driven treadmill ('Valiant'; Lode B.V., Groningen, Netherlands) to determine peak oxygen uptake ( $\mathrm{VO}_{2}$ peak), time to exhaustion and gas exchange threshold $\left(\mathrm{T}_{\mathrm{ge}}\right)$. Before the first exercise testing session, participants were familiarized with treadmill walking at various speeds $\left(2.0-6.0 \mathrm{~km} \mathrm{~h}^{-1}\right)$ and treadmill grades $(0-10 \%)$. Each subject's preferred walking speed was determined (at $1 \%$ grade). Preferred walking speed was used as the treadmill speed during all subsequent exercise tests and exercise training sessions. The incremental exercise test consisted of a 4 -min warm-up at $3.0 \mathrm{~km} \cdot \mathrm{h}^{-1}$ and $1 \%$ grade, followed by a speed increase every minute until the previously determined preferred walking speed was attained. Treadmill grade was then increased by $2 \%$ every minute until volitional fatigue or clinically significant signs or symptoms precluded further exercise. Cardiac rate and rhythm was monitored continuously using the 12 lead EKG and brachial artery blood pressure was measured by auscultation every 3 minutes during the incremental exercise test. Carbon dioxide output $\left(\mathrm{VCO}_{2}\right)$, oxygen uptake $\left(\mathrm{VO}_{2}\right)$, and expired minute ventilation $\left(\mathrm{V}_{\mathrm{E}}\right.$ BTPS) were measured breath-by-breath and averaged every 30 seconds using an open-circuit metabolic measurement system (Ultima CPX, Medical Graphics Corporation, St Paul, USA). The gas analyzers and pneumotachograph were calibrated before and after each incremental exercise test using precision reference gases and an independently calibrated 3-L calibration syringe (Hans Rudolph Inc., Kansas City, USA). The average of the two highest consecutive 30 -second values measured before volitional fatigue was used to determine peak gas exchange $\left(\mathrm{VO}_{2}, \mathrm{VCO}_{2}\right)$ values. $\mathrm{T}_{\mathrm{ge}}$ was determined using the simplified V-slope method [23]

\section{Exercise Training Program}

After recruitment, dependent variables were measured 6 weeks before commencing exercise training (acting as a control period for each subject) ( $-6 \mathrm{wk})$, immediately before starting the exercise training program $(0 \mathrm{wk})$, and after 6 and 12 weeks of exercise training. All participants exercised by walking on a motor driven treadmill for a total of 120 minute.week ${ }^{-1}$, either 2 or 4 times per week and at an intensity equivalent to their individual $\mathrm{T}_{\mathrm{ge}}$. Before commencing exercise training (0 wk), participants were again familiarized with walking on a motordriven treadmill. Participants completed a 3-minute warm-up and a 3 -minute cool-down at $3 \mathrm{~km} \cdot \mathrm{hr}{ }^{-1}$ and $1 \%$ grade for all exercise training sessions. Before each exercise training session, participants were fitted with a 5-lead EKG (X12+, Mortara Instrument Inc., Milwaukee, USA) to continuously monitor heart rate and rhythm. Heart rate and blood pressure were recorded every 5 minutes throughout all exercise training sessions.

Exercise training intensity was individually determined during the first exercise training session using breath-by-breath open-circuit spirometry, to determine $\mathrm{VO}_{2}$. While at each subject's preferred walking speed, treadmill grade was increased $1 \%$ every 3 minutes until steady-state $\mathrm{VO}_{2}$ matched their pre-determined $\mathrm{T}_{\mathrm{ge}}$. Based on maximal exercise test results obtained after 6 weeks of exercise training $(0-6 \mathrm{wk})$, exercise intensity was adjusted to maintain the same relative percent (100\%) of $\mathrm{T}_{\mathrm{ge}}$ for the remaining 6 weeks exercise training. Participants were instructed to continue their normal daily activities during both the 6 week control period and 12 week exercise-training program.

\section{Determination of ADMA}

Blood was collected from a prominent antecubital vein into EDTA blood collection tubes, which were immediately placed on a tube roller. Plasma was separated from whole blood within $30 \mathrm{~min}$ of collection and subsequently stored at $-80{ }^{\circ} \mathrm{C}$ until analyzed. Asymmetric dimethylarginine concentrations were assayed (in fasting plasma) using a commercially available enzyme-linked immunosorbent assay (ELISA) kit (Immundiagnostik AG, Bensheim, Germany). A Fluido microplate strip-washer (Anthos Labtec Instruments, Eugendorf, Austria) and Anthos 2020 absorbance reader (Anthos Labtec Instruments, Eugendorf, Austria) were utilized during all assays. The ELISA kit reported a sensitivity of $0.05 \mu \mathrm{mol} \cdot \mathrm{L}^{-1}$. The intra-assay coefficient of variation ranged from $5.3-7.7 \%$. Standards and controls provided with the kits were used in measurements.

\section{Statistical Analysis}

Results are reported as mean \pm standard error unless otherwise stated. Data were analyzed using a mixed design analysis of variance 
Citation: Serre KR, Simmonds MJ, Sabapathy S, Minahan CL, Gass GC (2011) Rapid Communication - Effect of Exercise Training on Asymmetric Dimethylarginine Concentration in Women Aged 65-74 years with Type 2 Diabetes. Endocrinol Metabol Syndrome S5:001. doi:10.4172/21611017.S5-001

Page 3 of 5

(ANOVA) with exercise group as the between-subject variable, and weeks $(0,6,12 \mathrm{wk})$ as the within-subject variable. All statistical differences were accepted at the $\mathrm{p}<0.05$ level. SPSS (SPSS Inc, Release 17.0, USA) was used for all statistical analyses.

\section{Results}

Of the fifteen individuals who started the exercise training program, fourteen individuals completed all repeated testing periods, blood draws, and exercise training sessions. No significant differences were found in any dependent variables before or after the 6-week intervention-free control period (-6 to $0 \mathrm{wk}$ ) (Table 1 ).

After 6 and 12-weeks of exercise training, there were no significant changes in body mass, BMI or waist-to-hip ratio. However, both systolic and diastolic blood pressure was significantly reduced after 12 weeks exercise training $(\mathrm{p}<0.05)$. No significant changes were found in other blood or lipid profile measurements after 12 weeks of exercise training ( $\mathrm{p}>0.05)$ (Table 1).

Twelve weeks of exercise training at an intensity equivalent to Tge (72-74\% $\mathrm{VO}_{2}$ peak) resulted in significant increases in time to exhaustion, absolute and relative $\mathrm{VO}_{2}$ peak, and $\mathrm{VO}_{2}$ at $\mathrm{T}_{\mathrm{ge}}(\mathrm{p}<0.05)$ (Table 1).

Plasma ADMA concentration was found to be significantly lower after 12-weeks exercise training from baseline measurements $(0 \mathrm{wk})(\mathrm{p}$ $<0.05$; Table 1; Figure 1).

\section{Discussion and Conclusions}

Our study found that 12 weeks of moderate-intensity exercise training (120 minutes per week at an intensity equivalent to individual $\mathrm{T}_{\mathrm{ge}}$ ) significantly lowered plasma ADMA concentration in women aged 65-74 years with type 2 diabetes. To our knowledge, this is the first study showing clinical evidence for exercise as a therapeutic intervention to lower ADMA concentration in older women with type 2 diabetes.

Participants in the present study had plasma ADMA concentrations consistent with those found in other studies of post-menopausal women [24]. In agreement with the present study, exercise-induced decreases in systemic ADMA concentration have been previously reported in different populations [19,21,24-27]. Moderate-intensity, supervised exercise has shown to decrease plasma ADMA concentration in individuals with elevated cardiovascular risk [19], including peripheral arterial disease [26], chronic heart failure [27], obesity [25], and type 1 diabetes [21]. Exercise training has also been shown to be ineffective in decreasing plasma ADMA [28]. Plasma ADMA concentration was unaffected by 2 months of unsupervised, home-based exercise training in heart failure patients [28]. ADMA concentration in this study was similar between patients and control participants, and normal ADMA concentrations might not have been further lowered by exercise. Plasma ADMA concentration was also found to be unaffected by 16 weeks of supervised exercise training in another cohort of heart failure patients [29]. However, the exercise dose may have been insufficient to elicit a decrease in ADMA concentration as the exercise mode (dancing) was performed in a group environment and music speed was used to moderate intensity according self-reported rating of perceived exertion [29]. Unlike the present study, exercise stimulus was not individualized nor linked to a physiological marker such as $\mathrm{T}_{\text {ge }}$.

In the present study, twelve weeks of moderate-intensity exercise training resulted in significant improvements in multiple measures of physiological functional capacity, including TE, $\mathrm{VO}_{2}$ peak, and $\mathrm{VO}_{2}$ at $\mathrm{T}_{\mathrm{ge}}$. These improvements were accompanied by significant decreases in both systolic and diastolic blood pressure (BP) following twelve weeks of exercise. The exercise-induced reductions in ADMA plasma concentration has been reported elsewhere [19,21,24-27], the mechanisms behind this process are not clear. ADMA's cardiovascular effects include vasoconstriction and increased blood pressure $[30,31]$

\begin{tabular}{|c|c|c|c|c|}
\hline & Week $-6(n=14)$ & Week $0(n=14)$ & Week $6(n=14)$ & Week $12(n=14)$ \\
\hline Age (yrs) & $68.9 \pm 0.7$ & $68.9 \pm 0.7$ & $68.9 \pm 0.7$ & $68.9 \pm 0.7$ \\
\hline Body Mass (kg) & $76.5 \pm 3.7$ & $75.9 \pm 3.8$ & $76.2 \pm 3.9$ & $76.4 \pm 3.8$ \\
\hline BMI $\left(\mathrm{kg} \cdot \mathrm{m}^{-2}\right)$ & $30.2 \pm 1.4$ & $30.0 \pm 1.4$ & $30.1 \pm 1.4$ & $30.1 \pm 1.4$ \\
\hline Waist/Hip Ratio & $0.86 \pm 0.02$ & $0.85 \pm 0.02$ & $0.86 \pm 0.02$ & $0.86 \pm 0.02$ \\
\hline \multicolumn{5}{|l|}{ Blood Pressure $(\mathrm{mmHg})$} \\
\hline $\mathrm{SBP}_{\text {rest }}$ & $134 \pm 3$ & $133 \pm 2$ & $130 \pm 3$ & $122 \pm 3^{*}$ \\
\hline $\mathrm{DBP}_{\text {rest }}$ & $76 \pm 2$ & $75 \pm 2$ & $70 \pm 2$ & $69 \pm 2^{*}$ \\
\hline \multicolumn{5}{|l|}{ Blood Profile } \\
\hline ADMA $\left(\mu \mathrm{mol} \cdot \mathrm{L}^{-1}\right)$ & $0.68 \pm 0.04$ & $0.71 \pm 0.02$ & $0.69 \pm 0.04$ & $0.62 \pm 0.03^{*}$ \\
\hline Homocysteine $\left(\mu \mathrm{mol} \cdot \mathrm{L}^{-1}\right)$ & $12.8 \pm 1.8$ & $11.6 \pm 1.3$ & $11.6 \pm 0.9$ & $11.6 \pm 0.8$ \\
\hline Glucose $\left(\mathrm{mmol} \cdot \mathrm{L}^{-1}\right)$ & $7.4 \pm 0.5$ & $7.5 \pm 0.5$ & $7.0 \pm 0.4$ & $7.2 \pm 0.4$ \\
\hline $\mathrm{HbA}_{1 \mathrm{C}}(\%)$ & $6.6 \pm 0.2$ & $6.6 \pm 0.2$ & $6.5 \pm 0.2$ & $6.7 \pm 0.2$ \\
\hline $\mathrm{HbA}_{1 \mathrm{C}}\left(\mathrm{mmol} \cdot \mathrm{mol}^{-1}\right)$ & $49 \pm 2$ & $49 \pm 2$ & $48 \pm 2$ & $50 \pm 2$ \\
\hline Insulin $\left(\mathrm{mU} \cdot \mathrm{L}^{-1}\right)$ & $11.3 \pm 2.3$ & $11.0 \pm 2.2$ & $12.9 \pm 2.6$ & $13.6 \pm 3.5$ \\
\hline \multicolumn{5}{|l|}{ Exercise Responses } \\
\hline TE (min) & $13.7 \pm 0.5$ & $13.9 \pm 0.4$ & $15.4 \pm 0.4^{*}$ & $16.4 \pm 0.4^{*}$ \\
\hline $\mathrm{VO}_{2}$ peak $\left(\mathrm{L} \cdot \mathrm{min}^{-1}\right)$ & $1.49 \pm 0.03$ & $1.45 \pm 0.04$ & $1.53 \pm 0.05$ & $1.59 \pm 0.05^{*}$ \\
\hline $\mathrm{VO}_{2}$ peak $\left(\mathrm{mL} \cdot \mathrm{kg}^{-1} \cdot \mathrm{min}^{-1}\right)$ & $20.0 \pm 0.9$ & $19.6 \pm 1.1$ & $20.5 \pm 0.9$ & $21.4 \pm 1.0^{*}$ \\
\hline Peak HR (b·min $\left.{ }^{-1}\right)$ & $143 \pm 3$ & $141 \pm 5$ & $144 \pm 4$ & $145 \pm 3$ \\
\hline$V_{E}$ peak $\left(L \cdot \mathrm{min}^{-1}\right)$ & $51.7 \pm 1.8$ & $50.8 \pm 2.4$ & $55.2 \pm 2.4^{*}$ & $54.2 \pm 2.5$ \\
\hline $\mathrm{VO}_{2} \mathrm{~T}_{\mathrm{ge}}\left(\mathrm{L} \cdot \mathrm{min}^{-1}\right)$ & $1.04 \pm 0.03$ & $1.01 \pm 0.04$ & $1.04 \pm 0.04$ & $1.12 \pm 0.05^{\star}$ \\
\hline Peak RER & $1.10 \pm 0.02$ & $1.11 \pm 0.03$ & $1.13 \pm 0.03$ & $1.07 \pm 0.02$ \\
\hline
\end{tabular}

BMI, body mass index; SBP, systolic blood pressure; DBP, diastolic blood pressure; $\mathrm{HbA}_{1}$, glycated hemoglobin; TE, time to exhaustion; VO ${ }_{2}$ eak, peak oxygen uptake; $\mathrm{HR}$, heart rate; $\mathrm{V}_{\mathrm{E}}$, ventilation; $\mathrm{VO}_{2} \mathrm{~T}_{\mathrm{ge}}$, oxygen uptake at gas-exchange threshold; $\mathrm{RER}$, respiratory exchange ratio. ${ }^{*} \mathrm{p}<0.05$, significantly different to pre-training $(0 \mathrm{wk})$

Table 1: Clinical characteristics of participating participants before (week -6) and after (week 0) 6 week intervention-free control period and after 6 and 12 weeks of exercise training (Mean \pm SEM). 
Citation: Serre KR, Simmonds MJ, Sabapathy S, Minahan CL, Gass GC (2011) Rapid Communication - Effect of Exercise Training on Asymmetric Dimethylarginine Concentration in Women Aged 65-74 years with Type 2 Diabetes. Endocrinol Metabol Syndrome S5:001. doi:10.4172/21611017.S5-001

Page 4 of 5

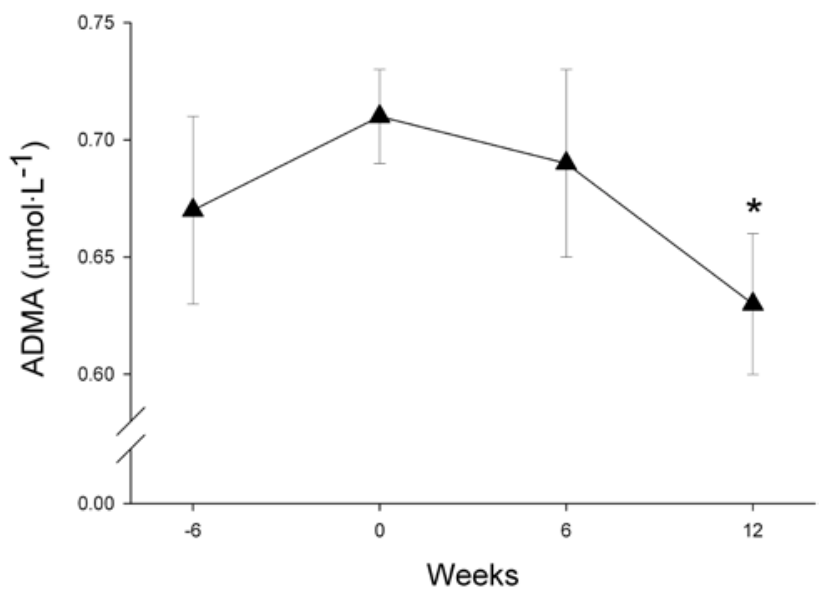

Data presented as mean \pm SEM. ${ }^{*}, p<0.05$, significantly different to pretraining $(0 \mathrm{wk})$.

Figure 1: Changes in plasma ADMA concentration $(\boldsymbol{\Delta})$ during 6 week control period (wk -6 to wk 0 ), and at 6 and 12 weeks exercise training (wk 0 to wk 6 to wk 12).

and may explain, in part, the significant reduction in BP seen in the present study after 12 weeks of exercise training. This argument may be strengthened research suggesting that exercise-induced increases in NO bioavailability may be due, at least in part, to decreases in ADMA concentration [20].

It has been hypothesized that regular exercise decreases ADMA concentration by upregulating DDAH-1, an enzyme involved in ADMA decomposition [21]. Recently, decreased ADMA concentration following exercise training has been shown to be accompanied by enhanced DDAH-1 mRNA gene-expression [25]. Upregulation of DDAH-1 and subsequent decreased ADMA concentration through regular exercise may be influenced by antioxidant status. Recent work has suggested that aging-associated oxidant/antioxidant imbalance promotes the formation cardiovascular risk factors, including ADMA, via increases in systemic oxidative stress in elderly individuals [32]. Unfortunately, no measure of oxidative stress was measured in the present study and these findings cannot be confirmed.

The significant decrease in plasma ADMA concentration in the present study was not accompanied by significant improvements in conventional markers of cardiovascular disease (CVD) risk factors, including homocysteine concentration. The reduction in plasma ADMA concentration may be independent of homocysteine and may act as a sensitive marker of CVD risk in medically well-managed patients who may not show more conventional signs of CVD risk.

A potential limitation in our study is the extrapolation of the results beyond the cohort of participants in the present study. While subject recruitment proved difficult due to the significant time requirements of the exercise training program and the use of a repeated measure design, the modest sample size used in our study needs to be acknowledged as a potential limitation, but mitigated by the present experimental design.

In conclusion, 12 weeks moderate-intensity exercise training (walking) can significantly reduce circulating plasma ADMA concentration and systolic and diastolic blood pressure in women aged 65-74 years with type 2 diabetes. Our study suggests that the decreased incidence of vascular disease associated with regular exercise in type 2 Diabetes may be, in part, explained by decreased circulating plasma ADMA concentration.

\section{Acknowledgements}

This study was supported in part by the National Health and Medical Research Council of Australia (Grant No. 376601) and Bond University. The authors thank Dr. Laurie Kelly for supervising the incremental exercise tests; Mrs. Tracey Norling, Ms. Siri Lauluten, Mr. Matthew Haycock and Mr. Rhys Christy for assisting in data collection; and Diabetes Australia Queensland for their help in recruitment efforts.

\section{Duality of Interest}

The authors declare that there is no duality of interest associated with this manuscript.

\section{References}

1. Kannel WB, McGee DL (1979) Diabetes and cardiovascular disease. The Framingham study. JAMA 241: 2035-2038.

2. Yamagishi S, Imaizumi T (2005) Diabetic vascular complications: pathophysiology, biochemical basis and potential therapeutic strategy. Curr Pharm Des 11: 2279-2299.

3. Schalkwijk CG, Stehouwer CD (2005) Vascular complications in diabetes mellitus: the role of endothelial dysfunction. Clin Sci (Lond) 109: 143-159.

4. Kawata T, Daimon M, Hasegawa R, Teramoto K, Toyoda T, et al. (2005) Serum asymmetric dimethylarginine as a marker of coronary microcirculation in patients with non-insulin dependent diabetes mellitus: correlation with coronary flow reserve. Heart 91: 1607-1608.

5. Lin KY, Ito A, Asagami T, Tsao PS, Adimoolam S, et al. (2002) Impaired nitric oxide synthase pathway in diabetes mellitus: role of asymmetric dimethylarginine and dimethylarginine dimethylaminohydrolase. Circulation 106: 987-992.

6. Vallance P, Leiper J (2004) Cardiovascular biology of the asymmetric dime thylarginine:dimethylarginine dimethylaminohydrolase pathway. Arterioscler Thromb Vasc Biol 24 1023-1030.

7. Vallance P, Leone A, Calver A, Collier J, Moncada S (1992) Endogenous dimethylarginine as an inhibitor of nitric oxide synthesis. J Cardiovasc Pharmacol 12: 60-62.

8. Böger RH, Bode-Böger SM, Szuba A, Tsao PS, Chan JR, et al. (1998) Asymmetric dimethylarginine (ADMA): a novel risk factor for endothelial dysfunction: its role in hypercholesterolemia. Circulation 98: 1842-1847.

9. Surdacki A, Nowicki M, Sandmann J, Tsikas D, Boeger RH, et al. (1999) Reduced urinary excretion of nitric oxide metabolites and increased plasma levels of asymmetric dimethylarginine in men with essential hypertension. $J$ Cardiovasc Pharmacol 33: 652-658.

10. Vallance P, Leone A, Calver A, Collier J, Moncada S (1992) Accumulation of an endogenous inhibitor of nitric oxide synthesis in chronic renal failure. Lancet 339: $572-575$

11. Schnabel R, Blankenberg S, Lubos E, Lackner KJ, Rupprecht HJ, (2005) Asymmetric dimethylarginine and the risk of cardiovascular events and death in patients with coronary artery disease: results from the AtheroGene Study. Circ Res 97 e53-59.

12. Miyazaki H, Matsuoka H, Cooke JP, Usui M, Ueda S, et al. (1999) Endogenous nitric oxide synthase inhibitor: a novel marker of atherosclerosis. Circulation 99 1141-1146.

13. Abbasi F, Asagmi T, Cooke JP, Lamendola C, McLaughlin T, (2001) Plasma concentrations of asymmetric dimethylarginine are increased in patients with type 2 diabetes mellitus. Am J Cardiol 88: 1201-1203.

14. Malecki MT, Undas A, Cyganek K, Mirkiewicz-Sieradzka B, Wolkow P, et al. (2007) Plasma asymmetric dimethylarginine (ADMA) is associated with retinopathy in type 2 diabetes. Diabetes Care 30: 2899-2901.

15. S. Yamagishi S, Ueda S, Nakamura K, Matsui T, Okuda S (2008) Role of asymmetric dimethylarginine (ADMA) in diabetic vascular complications. Cur Pharm Des 14 2613-2618.

16. de Jager J, Dekker JM, Kooy A, Kostense PJ, Nijpels G, et al. (2006) Endothelial dysfunction and low-grade inflammation explain much of the excess cardiovascular mortality in individuals with type 2 diabetes: the Hoorn Study. Arterioscler Thromb Vasc Biol 26 1086-1093.

17. Maiorana A, O'Driscoll G, Cheetham C, Dembo L, Stanton K, (2001) The effect of combined aerobic and resistance exercise training on vascular function in type 2 diabetes. J Am Coll Cardiol 38: 860-866. 
Citation: Serre KR, Simmonds MJ, Sabapathy S, Minahan CL, Gass GC (2011) Rapid Communication - Effect of Exercise Training on Asymmetric Dimethylarginine Concentration in Women Aged 65-74 years with Type 2 Diabetes. Endocrinol Metabol Syndrome S5:001. doi:10.4172/21611017.S5-001

Page 5 of 5

18. Okada S, Hiuge A, Makino H, Nagumo A, Takaki H, (2010) Effect of exercise intervention on endothelial function and incidence of cardiovascular disease in patients with type 2 diabetes. J Atheroscler Thromb 17: 828-833.

19. Richter B, Niessner A, Penka M, Grdić M, Steiner S, et al. (2005) Endurance training reduces circulating asymmetric dimethylarginine and myeloperoxidase levels in persons at risk of coronary events. Thromb Haemost 94: 1306-1311.

20. Gomes VA, Casella-Filho A, Chagas AC, Tanus-Santos JE (2008) Enhanced concentrations of relevant markers of nitric oxide formation after exercise training in patients with metabolic syndrome. Nitric Oxide 19: 345-350.

21. Mittermayer F, Pleiner J, Krzyzanowska K, Wiesinger GF, Francescon M, (2005) Regular physical exercise normalizes elevated asymmetrical dimethylarginine concentrations in patients with type 1 diabetes mellitus. Wien Klin Wochenschr 117: 816-820.

22. Folstein MF, Folstein SE, McHugh PR (1975) «Mini-mental state». A practical method for grading the cognitive state of patients for the clinician. J Psychiatr Res 12: 189-198.

23. Schneider DA, Phillips SE, Stoffolano S (1993) The simplified V-slope method of detecting the gas exchange threshold. Med Sci Sports Exerc 25: 1180-1184.

24. Sydow K, Fortmann SP, Fair JM, Varady A, Hlatky MA, (2010) Distribution of asymmetric dimethylarginine among 980 healthy, older adults of different ethnicities. Clin Chem 56: 111-120.

25. Hanssen H, Nickel T, Drexel V, Hertel G, (2011) Emslander I, Exercise-induced alterations of retinal vessel diameters and cardiovascular risk reduction in obesity. Atherosclerosis 216:433-439.
26. Schlager O, Giurgea A, Schuhfried O, Seidinger D, Hammer A, et al. (2011) Exercise training increases endothelial progenitor cells and decreases asymmetric dimethylarginine in peripheral arterial disease: A randomized controlled trial. Atherosclerosis 217: 240-248.

27. Tsarouhas K, Karatzaferi C, Tsitsimpikou C, Haliassos A, Kouretas D, et al. (2011) Effects of walking on heart rate recovery, endothelium modulators and quality of life in patients with heart failure. Eur J Cardiovasc Prev Rehabi 18:594-600.

28. Niebauer J, Clark AL, Webb-Peploe KM, Böger R, Coats AJ (2005) Homebased exercise training modulates pro-oxidant substrates in patients with chronic heart failure. Eur J Heart Fail 7: 183-188.

29. Seljeflot I, Nilsson BB, Westheim AS, Bratseth V, Arnesen H (2011) The $\mathrm{L}$-arginine-asymmetric dimethylarginine ratio is strongly related to the severity of chronic heart failure. No effects of exercise training. J Card Fail 17: 135-142.

30. Achan V, Broadhead M, Malaki M, Whitley G, Leiper J, et al. (2003) Asymmetric dimethylarginine causes hypertension and cardiac dysfunction in humans and is actively metabolized by dimethylarginine dimethylaminohydrolase. Arterioscler Thromb Vasc Biol 23: 1455-1459.

31. Kielstein JT, Impraim B, Simmel S, Bode-Böger SM, Tsikas D, et al. (2004) Cardiovascular effects of systemic nitric oxide synthase inhibition with asymmetrical dimethylarginine in humans. Circulation 109: 172-177.

32. Fabian E, Bogner M, Elmadfa I (2011) Age-related modification of antioxidan enzyme activities in relation to cardiovascular risk factors. Eur J Clin Invest 1111: 1365-2362.
This article was originally published in a special issue, Diabetes \& Types handled by Editor(s). Dr. Teik Chye Ooi, University of Ottawa, Canada; Dr. Panagiotis Anagnosti, Hippokration Hospital, Greece
Submit your next manuscript and get advantages of OMICS Group submissions

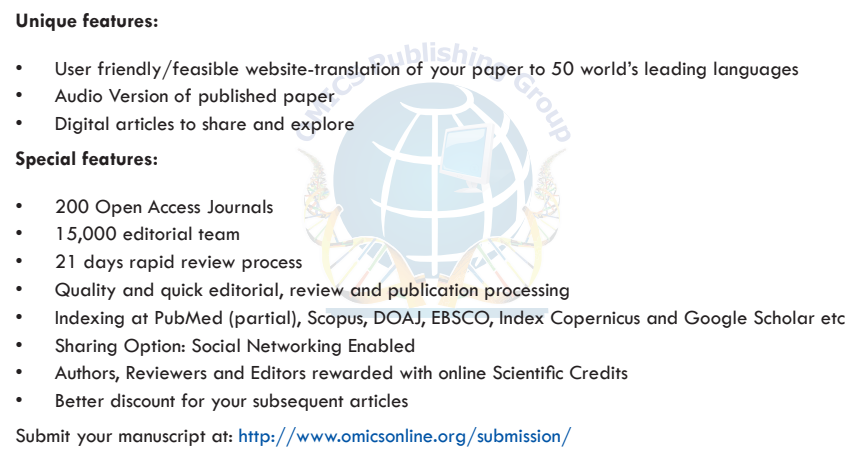

